\title{
Effects of Asymmetries in the Rotor and Flexible Joint of a Dual-Spin Spacecraft
}

\author{
B. N. AgrawaL* \\ COMSAT Laboratories, Clarksburg, Md.
}

\section{$T$}

HIS paper concerns the effects of inequalities in the transverse inertias and stiffnesses of the flexible joint of a dual-spin spacecraft on its stability and nutation frequency.

\section{Contents}

Most past investigations of dual-spin spacecraft have assumed that the platform and rotor are symmetric and rigidly connected. Recently, Cretcher and Mingori, ${ }^{1}$ Wenglarz, ${ }^{2}$ Willems, ${ }^{3}$ and Bainum $^{4}$ have considered the effects of flexibility in different parts of dual-spin spacecraft. Scher and Farrenkopf ${ }^{\circ}$ have considered the effects of an asymmetric rotor rigidly connected to the platform. However, the effects of unsymmetric transverse inertias in a dual-spin spacecraft with a flexible joint have not been published. They have been rigorously investigated in rotors and gyroscopes by Brosens and Crandall ${ }^{6}$ and by Foote, Poritsky, and Slade. ${ }^{7}$ This paper presents the results of an investigation of these effects on the attitude motion of dual-spin spacecraft. It includes only those cases for which the equations of motion can be transformed into linear equations without periodic coefficients.

The model, shown in Fig. 1, consists of a spinning rotor which is connected to a nonspinning platform by a flexible joint. The axes $X_{0} Y_{0} Z_{0}$ are fixed in space with the origin at the $\mathrm{CM}$ of the system. The axes $X_{2} Y_{2} Z_{2}$ are the principal inertia axes of the platform and are described with respect to axes $X_{0} Y_{0} Z_{0}$ by rotations $\theta_{x}$ about the $X_{0}$ axis and $\theta_{y}$ about the $Y_{1}$ axis. The orientations of $X_{4} Y_{4} Z_{4}$, where $Z_{4}$ is the drive axis of the rotor, are described by rotations $\phi_{x}$ about the $X_{2}$ axis and $\phi_{y}$ about the $Y_{3}$ axis. Axes $X_{4}{ }^{1} Y_{4}{ }^{1} Z_{4}{ }^{1}$, which will be the principal inertia axes for a balanced rotor, are fixed in the rotor and rotating at the spin rate about the $Z_{4}$ axis. For an unbalanced rotor, the principal axes are $X_{6} Y_{6} Z_{6}$, which are defined by rotations $\Omega_{x}$ about the $X_{4}{ }^{1}$ axis and $\Omega_{y}$ about the $Y_{5}$ axis. The center of mass offset of the rotor is $\varepsilon$. Both the rotor and the platform are assumed to have unequal transverse inertias and the rotor is assumed to be dynamically and statically unbalanced. The equations of motion of this system are obtained by using Lagrangian formulation. Two cases are considered for unsymmetrical stiffness.

Case I: Inequality in stationary stiffness. In this case, the stiffness inequality in the joint is associated with the platform. The equations of motion are linear gyroscopic equations with periodic coefficients contributed by the inequality in the transverse inertias of the rotor. For a dual-spin spacecraft with symmetric rotor and unsymmetric platform and stiffness, the equations of motion

Received August 17, 1973; synoptic received March 20, 1974. Full paper available from National Technical Information Service. Springfield, Va., 22151, as N74-22499 at the standard price (available upon request). This Synoptic is based upon work performed in COMSAT Laboratories under the sponsorship of the International Telecommunications Satellite Orgarization (INTELSAT). Views expressed in this Synoptic are not necessarily those of INTELSAT.

Index category: Spacecraft Attitude Dynamics and Control.

* Member of the Technical Staff. Member AIAA. are linear gyroscopic equations. The characteristic equation, which gives the natural frequencies, is obtained for this system. For the symmetric platform and stiffness, the characteristic equation is simplified as follows:

$\lambda^{3}\left(I_{p r}{ }^{2}-\bar{I}_{t r} \bar{I}_{p}\right)+\lambda^{2} \omega \bar{I}_{p} I_{z r}+\lambda K\left(\bar{I}_{p}+\bar{I}_{t r}+2 I_{p r}\right)-K I_{z r} \omega=0$

where $\lambda=$ natural frequency; $\omega=$ spin rate of the rotor; $m_{p}=$ platform mass; $l_{p}=$ distance between the joint and the platform $\mathrm{CM} ; l_{r}=$ distance between the joint and the platform $\mathrm{CM} ; I_{p}=$ mean transverse inertia of the platform; $I_{t r}=$ mean transverse inertia of the rotor; $I_{z r}=$ rotor axial inertia; $m=$ reduced mass $=\left(m_{r} m_{p}\right) /\left(m_{r}+m_{p}\right) ; \quad \bar{I}_{t r}=I_{t r}+m l_{r}{ }^{2} ; \quad \bar{I}_{p}=$ $I_{p}+m l_{p}^{2} ; I_{p r}=m l_{p} l_{r} ;$ and $K=$ stiffness of the joint

For the parameters of a spacecraft similar to INTELSAT IV Fig. 2 shows the three natural frequencies as functions of the spin rate of the rotor. The first natural frequency is the nutation frequency, which is slightly lower than that obtained from rigid body analysis. The first structural frequency represents reverse precession and the second represents forward precession. In the

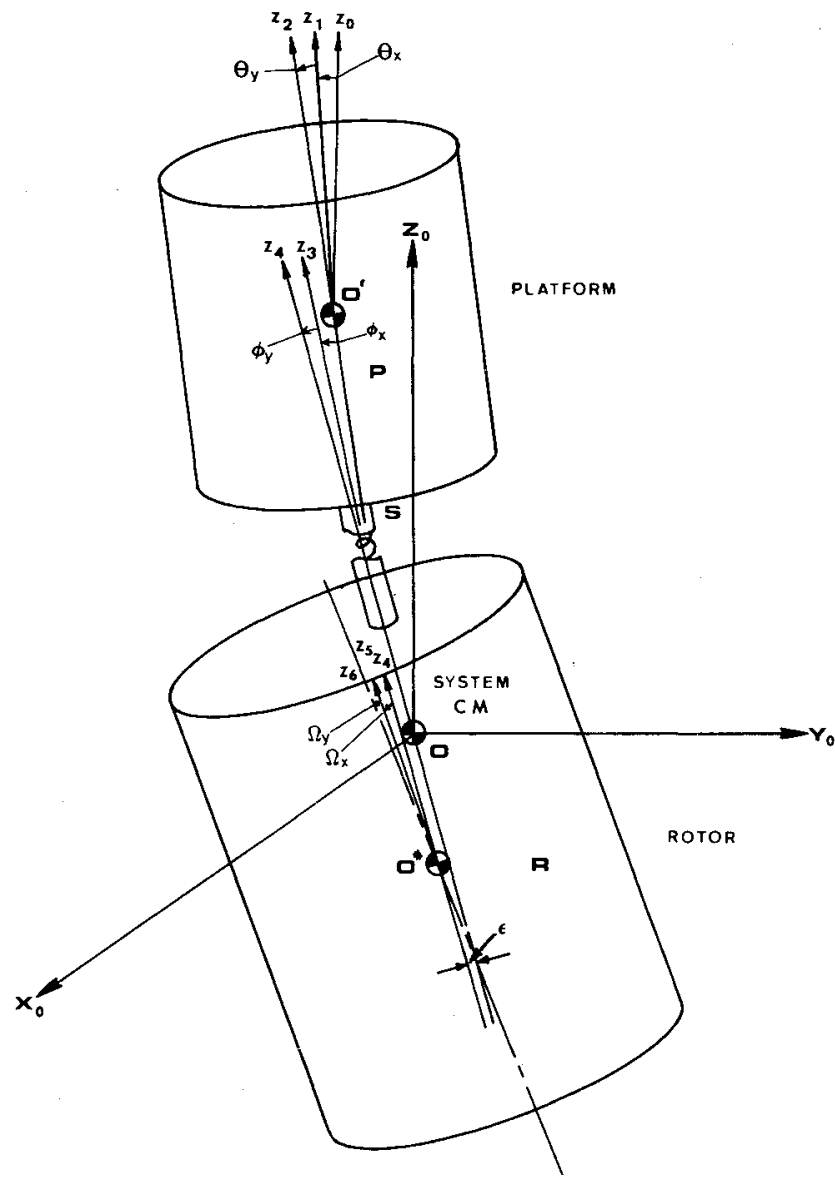

Fig. 1 Dual-spin satellites. 


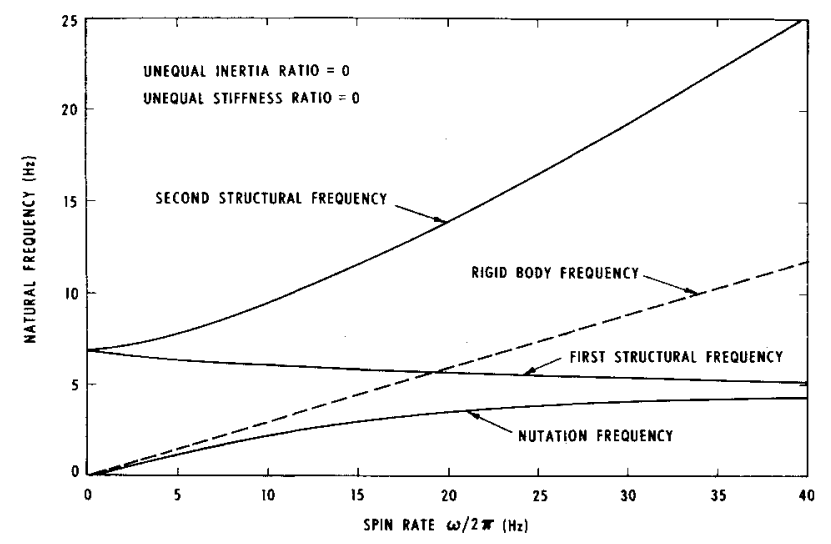

Fig. 2 Nutation frequency and other structural resonances for a symmetric spacecraft.

paper the mode shapes are obtained for an unsymmetric platform with inequality factor, $\alpha_{p}$, of 0.2 , and symmetric stiffness. Both platform and rotor have elliptical motion. In rotating coordinates, fixed with the rotor, the rotor motion, $\bar{\psi}_{x}$ and $\psi_{y}$, has two frequency terms. For a symmetric platform the motion will be circular and in rotating coordinates the rotor will have only one frequency term. The motion is found to be stable for all parameters considered.

Case II: Inequality in rotating stiffness. In this case, the stiffness inequality in the joint is associated with the rotor. The equations of motion in inertial coordinates are linear gyroscopic equations with periodic coefficients caused by inequalities in the rotor transverse inertias and joint stiffness. Transforming these equations into rotating coordinates, rotating at the spin rate of the rotor about the $Z$ axis, results in linear gyroscopic equations with periodic coefficients caused by inequality in the transverse inertias of the platform. For a balanced dual-spin spacecraft with symmetric platform and unsymmetric rotor and stiffness, the equations of motion are

$$
\begin{aligned}
& {\left[\begin{array}{cccc}
\bar{I}_{p} & 0 & I_{p r} & 0 \\
0 & \bar{I}_{p} & 0 & I_{p r} \\
I_{p r} & 0 & \bar{I}_{t r}+\sigma & 0 \\
0 & I_{p r} & 0 & \bar{I}_{t r}-\sigma
\end{array}\right]\left\{\begin{array}{c}
\ddot{\bar{\theta}}_{x} \\
\ddot{\bar{\theta}}_{y} \\
\ddot{\bar{\psi}}_{x} \\
\ddot{\bar{\psi}}_{y}
\end{array}\right\}+} \\
& {\left[\begin{array}{cccc}
0 & -2 \omega \bar{I}_{p} & 0 & -2 \omega I_{p r} \\
2 \omega \bar{I}_{p} & 0 & 2 \omega I_{p r} & 0 \\
0 & -2 \omega I_{p r} & 0 & -2 \omega \bar{I}_{t r} \\
2 \omega I_{p r} & 0 & 2 \omega \bar{I}_{t r} & 0
\end{array}\right]\left\{\begin{array}{c}
\dot{\bar{\theta}}_{x} \\
\dot{\bar{\theta}}_{y} \\
\dot{\bar{\psi}}_{x} \\
\dot{\bar{\psi}}_{y}
\end{array}\right\}+} \\
& {\left[\begin{array}{cccc}
K_{x}-\omega^{2} \bar{I}_{p} & 0 & -\left(K_{x}+\omega^{2} I_{p r}\right) & 0 \\
0 & K_{y}-\omega^{2} \bar{I}_{p} & 0 & -\left(K_{y}+\omega^{2} I_{p r}\right) \\
-\left(K_{x}+\omega^{2} I_{p r}\right) & 0 & K_{x}-\omega^{2}\left(\bar{I}_{t r}\right. & 0 \\
& & \left.-\sigma-I_{z r}\right) & 0 \\
0 & -\left(K_{y}+\omega^{2} I_{p r}\right) & 0 & K_{y}-\omega^{2}\left(\bar{I}_{t r}\right. \\
& & & \left.+\sigma-I_{z r}\right)
\end{array}\right] \times} \\
& \left\{\begin{array}{l}
\bar{\theta}_{x} \\
\bar{\theta}_{y} \\
\bar{\psi}_{x} \\
\bar{\psi}_{y}
\end{array}\right\}=0
\end{aligned}
$$

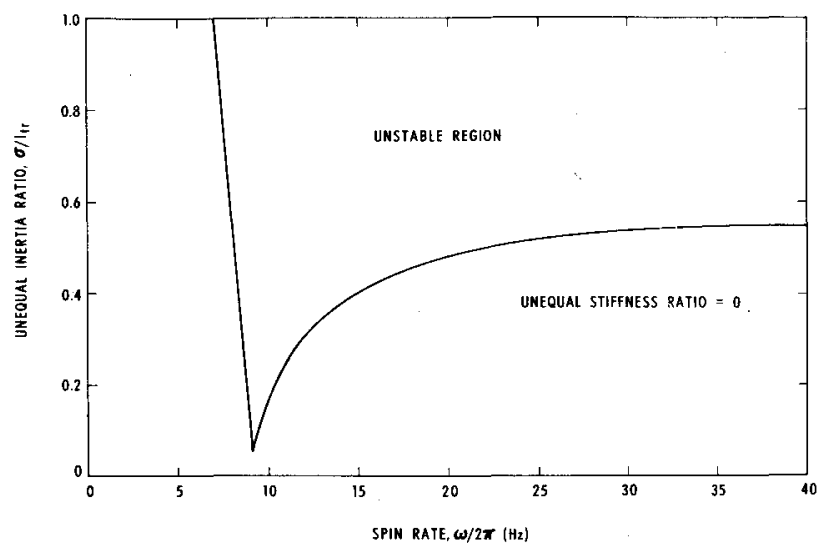

Fig. 3 Unstable region of motion for an unsymmetric rotor and symmetric platform.

where $K_{x}, K_{y}=$ rotational stiffnesses of the joint in directions $x$ and $y$, respectively, and $\sigma=$ half of the difference between transverse inertias, $\left(I_{x r}-I_{y r}\right) / 2$.

In Eq. (2), $\bar{\theta}_{x}, \bar{\theta}_{y}$, and $\bar{\psi}_{x}, \bar{\psi}_{y}$ are the rotational angles of the platform and rotor, respectively, in rotating coordinates. Stability regions are obtained by determining the eigenvalues of the characteristics matrix of Eq. (2) for parameters similar to those of INTELSAT IV. For unstable regions the real parts of the eigenvalues will be positive, resulting in an exponential increase in the amplitude of nutation. Figure 3 shows the unstable region for an unsymmetric rotor with symmetric stiffness.

In the paper, similar unstable regions are obtained for a symmetric rotor with unsymmetric stiffness. From this unstable region, it can be seen that with increasing spin rate the motion is initially stable. It becomes unstable for a certain spin rate and then becomes stable again. It should be noted that for the INTELSAT IV operating spin rate $(0.833 \mathrm{~Hz})$ and a rotor transverse inertia inequality factor of 0.0035 , the spacecraft is well within the stable region.

In summary, the nutation frequency of a dual-spin spacecraft predicted by a rigid body analysis is slightly higher than the actual nutation frequency calculated by including the flexibility of the joint in the analysis. The flexibility of the joint contributes rotational structural frequencies and unstable regions which are not predicted by the rigid body analysis.

\section{References}

${ }^{1}$ Cretcher, C. K. and Mingori, D. L., "Nutation Damping and Vibration Isolation in a Flexible Coupled Dual-Spin Spacecraft," Journal of Spacecraft and Rockets, Vol. 8, No. 8, Aug. 1971, pp. 817-823.

${ }^{2}$ Wenglarz, R. A., "Dynamically Unbalanced Dual-Spin Space Stations with Rigid or Low-Coupling Interconnections," Journal of Spacecraft and Rockets, Vol. 8, No. 10, Oct. 1971, pp. 1032-1037.

${ }^{3}$ Willems, P. Y., "Effect of Bearing Flexibility on Dual-Spin Spacecraft Attitude Stability," Journal of Spacecraft and Rockets, Vol. 9, No. 8, Aug. 1972, pp. 587-591.

${ }^{4}$ Bainum, P. M., Fuechsel, P. G., and Fedor, J. V., "Stability of a Dual-Spin Spacecraft with a Flexible Momentum Wheel," Journal of Spacecraft and Rockets, Vol. 9, No. 9, Sept. 1972, pp. 640-646.

${ }^{5}$ Scher, M. P. and Farrenkopf, R. L., "Dynamic Trap States of Dual-Spin Spacecraft," AIAA Journal, to be published.

${ }^{6}$ Brosens, P. J. and Crandall, S. H. "Whirling of Unsymmetrical Rotors," Journal of Applied Mechanics, Vol. 28, Sept. 1961, pp. 355-362.

${ }^{7}$ Foote, W. R., Poritsky, H., and Slade, J. J., Jr., "Critical Speeds of a Rotor with Unequal Shaft Flexibilities, Mounted in Bearings of Unequal Flexibilities," Transactions of the ASME: Journal of Applied Mechanics, Vol. 10, 1943, pp. A-77-A-84. 